\title{
SEMIINVASIVE ASPERGILLOSIS: A DIAGNOSIS TO CONSIDER IN A SEEMINGLY IMMUNOCOMPETENT PATIENT WITH A PULMONARY NODULE, HAEMOPTYSIS AND STRUCTURAL LUNG DISEASE
}

\author{
Francisco José Fernández-Fernández ${ }^{1}$, Marcelino del Castillo-Fraile ${ }^{2}$, María del Mar Used-Aznar ${ }^{3}$
}

Hospital Arquitecto Marcide, Ferrol. A Coruňa, Spain: Department of Internal Medicine'1, Department of Radiology², Department of Pathology ${ }^{3}$

Summary: Semiinvasive pulmonary aspergillosis is an indolent form of pulmonary aspergillosis, which is seen mainly in patients who are mildly immunocompromised with underlying chronic lung diseases. This syndrome is rare, and the available literature is based on case reports and small case series. We describe here a patient with a semiinvasive aspergillosis associated with a probable idiopathic pulmonary fibrosis.

Key words: Aspergillus; Aspergillosis, Hemoptysis; Pulmonary nodule

\section{Introduction}

Semiinvasive aspergillosis, also known as chronic necrotizing aspergillosis, is an indolent infectious process of the lung parenchyma secondary to local invasion by Aspergillus species (5). Patients frequently present with cough, low grade fever, and haemoptysis. Interpretation of radiologic studies, in this entity, may be complicated by the presence of concomitant lung disease, since this is the setting in which semiinvasive aspergillosis usually occurs $(2,3,4)$. The yield of bronchoalveolar lavage and transbronchial biopsy specimens is relatively poor for locally invasive aspergillosis, and thus, confirmation of the diagnosis is difficult $(4,5)$. We report here a patient, seemingly immunocompetent, with semiinvasive pulmonary aspergillosis who presented with haemoptysis and a pulmonary nodule.

\section{Case report}

In November, 2007, a 67-year-old man was admitted to the hospital because of a stroke with secondary partial seizures. The patient was a farmer with no history of smoking, or use of steroids, and he had progressive dyspnea on exertion. During his hospitalization, he experienced several limited episodes of coughing with blood-streaked sputum. A chest radiograph showed a bilateral interstitial disease, and a CT scan of the thorax revealed, among other findings, a nodular lesion with tiny cavitations in the left lower lobe (Fig. 1, left). He was discharged from hospital and referred to the outpatient pulmonary clinic for the evaluation of his respiratory process. His treatment at discharge included aspirin, valproate and omeprazole. In the clinic, a bronchoscopy with transbronchial biopsy and bronchoalveolar lavage (BAL) was performed. BAL fluid cultures were negative, and the transbronchial biopsy revealed a chronic inflammatory infiltrate with interstitial fibrosis. The results of routine hematologic, blood chemical, and enzyme tests, including the value for angiotensin-converting enzyme, were normal. An analysis of arterial blood while the patient was breathing ambient air revealed a $\mathrm{pH}$ of 7.49 , a partial pressure of carbon dioxide of $33 \mathrm{~mm} \mathrm{Hg}$, and a partial pressure of oxygen of $78 \mathrm{~mm} \mathrm{Hg}$. Immunoglobulin levels and serum protein electrophoresis showed no abnormalities. No study of granulocyte function was performed. Hypersensitivity screening for pneumonitis demonstrated a normal IgE level, and tests for antibodies to a standard panel of inhaled aeroallergens (Thermophilic actinomycetes) were negative. Tests for the presence of rheumatoid factor, antinuclear antibodies, and antineutrophil cytoplasmic autoantibodies were negative. The tuberculin skin test was negative. Pulmonary function tests showed a mildly decreased carbon monoxide diffusion capacity. Therapy with deflazacort was begun. Two months after initial presentation, and one month after starting deflazacort therapy, he was referred to our Department because of haemoptysis. Sputum samples were negative for culture. A CT scan was repeated, showing that the pulmonary nodule had evolved to a solid mass within a cavity, with thickening of the adjacent pleura, typical findings of an aspergilloma (Fig. 1, right). A CT scan-guided transthoracic needle biopsy of the cavitary lesion was carried out, which demonstrated numerous septate hyphae morphologically consistent with Aspergillus (Fig. 2). The 

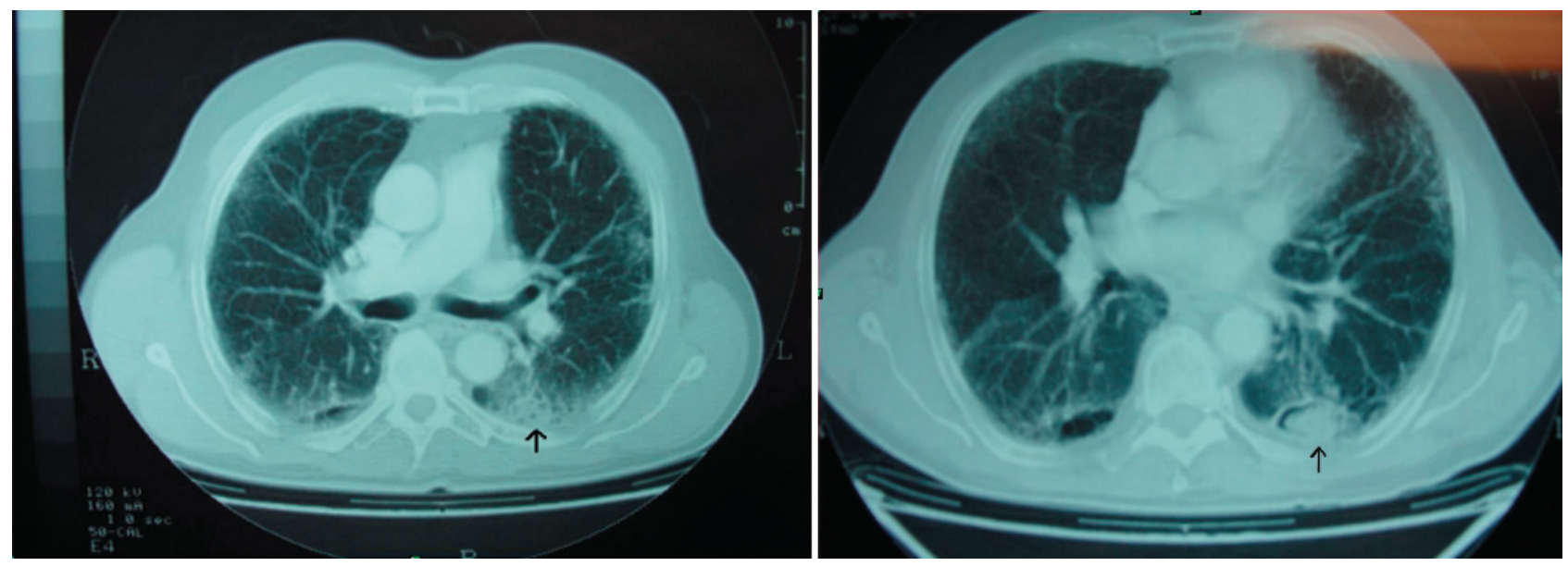

Fig. 1: Left - CT scan of the lung showing, among other findings, a nodule (arrow) with tiny cavitations in the left lower lobe. Right - CT scan of the thorax showing a solid mass separated from the wall of the cavity by an airspace, resulting in the characteristic air crescent sign. There is marked pleural thickening sorrounding the cavity.

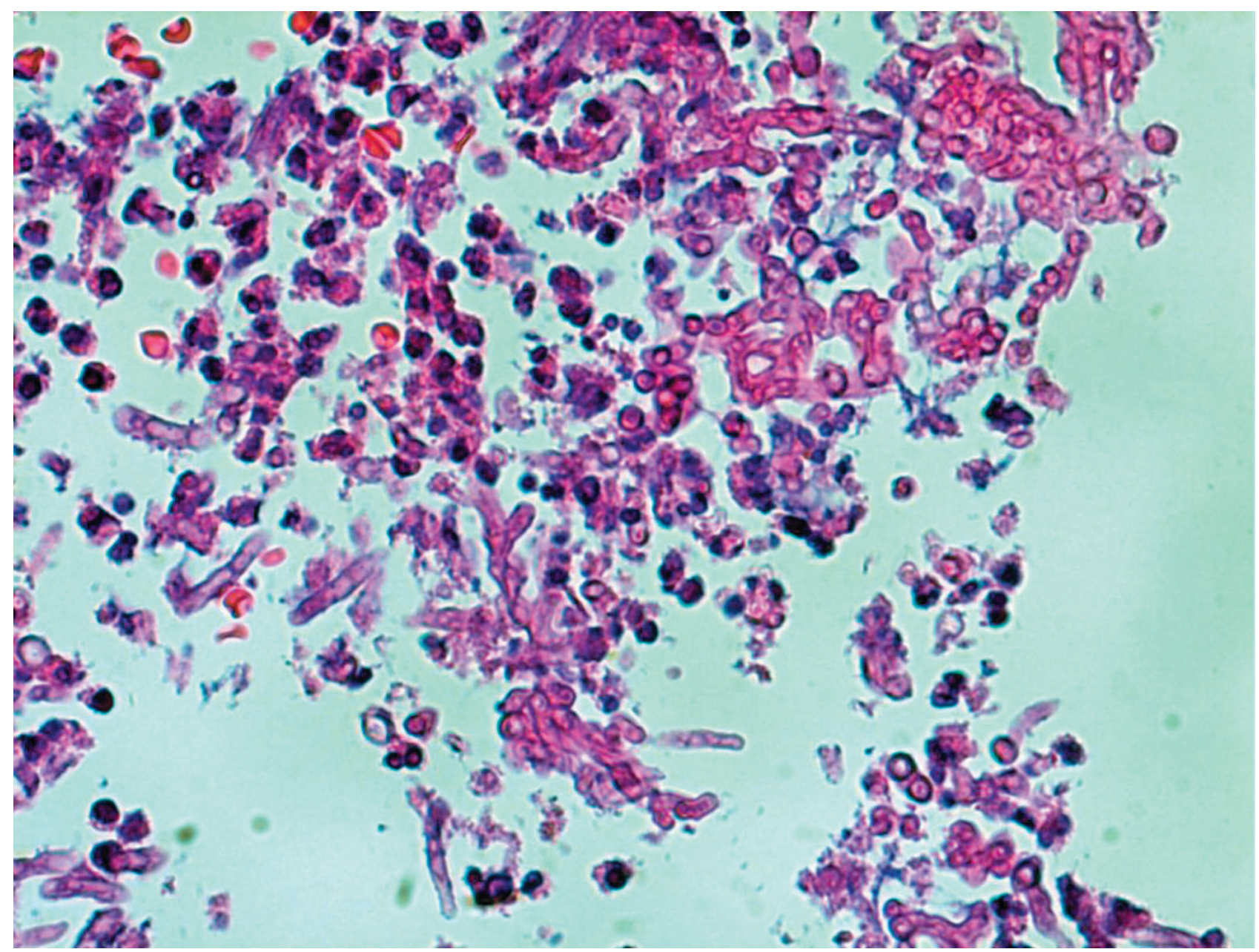

Fig. 2: Histopathology of the transthoracic needle biopsy of the pulmonary lesion showing numerous septate hyphae morphologically consistent with Aspergillus. 
culture was positive for Aspergillus fumigatus, and the patient's serum was positive for Aspergillus precipitins. He was started on itraconazole, with rapid reduction and withdrawal of steroids. A follow-up high resolution CT scan of the thorax, two months after starting itraconazole therapy, showed no change in the size of the aspergilloma, with radiological features typical of idiopathic pulmonary fibrosis.

\section{Discussion}

Semiinvasive aspergillosis is a indolent form of pulmonary aspergillosis, which is seen mainly in patients who are mildly immunocompromised with underlying chronic lung diseases $(2,3,4)$. The yield of bronchoalveolar lavage and transbronchial biopsy specimens is relatively poor, and a thoracoscopic or open-lung biopsy is rarely performed in these patients. Confirmation of the diagnosis is thus difficult, and delayed diagnosis is common (4).

In our patient, and in the context of pulmonary Aspergillus infection, the findings of the initial CT scan with a pulmonary nodule resembled the radiological findings of an invasive or semiivasive pulmonary aspergillosis (1). This farmer did not have any recognized underlying immune incompetence when he presented with stroke and the indolent clinical course would support a diagnosis of semiinvasive aspergillosis. On the other hand, and without having completed the study of the pulmonary nodule, he was mistakenly treated with steroids after the result of the transbronchial biopsy. In spite of that, the evolution was relatively benign with the formation of an aspergilloma. Presumably, the slowly progressive nature of his infection was a function of the host immune response, which was enough to barely hold the organism in check, but not to completely kill it. So then, it is important to take into account the possibility of pulmonary Aspergillus infection in seemingly immunocompetent patients, with underlying chronic lung diseases, who present with haemoptysis and a pulmonary nodule.

\section{Acknowledgment}

We thank to Mr. Diego Villares Fernández for his assistance in the composition of the figures.

\section{References}

1. Franquet T, Müller NL, Giménez A, Guembe P, de la Torre J, Bagué S. Spectrum of pulmonary aspergillosis: Histologic, clinical, and radiologic findings. Radiographics 2001;21:825-37.

2. Gefter WB, Weingrad TR, Epstein DM, Ochs RH, Miller WT. „Semi-invasive“ pulmonary aspergillosis: a new look at the spectrum of Aspergillus infections of the lung. Radiology 1981;140:313-21.

3. Kim SY, Lee KS, Han J, et al. Semiinvasive pulmonary aspergillosis: CT and pathologic findings in six patients. AJR Am J Roentgenol 2000;174:795-8.

4. Saraceno JL, Phelps DT, Ferro TJ, Futerfas R, Schwartz DB. Chronic necrotizing pulmonary aspergillosis. Chest 1997;112:541-8

5. Zmeili OS, Soubani AO. Pulmonary aspergillosis: a clinical update. Q J Med 2007;100:317-34

Received: 17/02/2009.

Accepted: 04/09/2009.

\section{Corresponding author:}

Dr. Francisco J. Fernández-Fernández, Department of Internal Medicine, Hospital Arquitecto Marcide, Ferrol 15405, Spain; e-mail: fjf-fernandez@terra.es 